ARTICLE

\title{
Structure units oriented approach towards collective synthesis of sarpagine-ajmaline-koumine type alkaloids
}

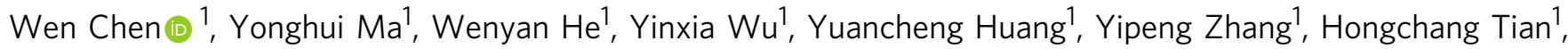
Kai Wei ${ }^{1}$, Xiaodong Yang (ib) ${ }^{1 凶} \&$ Hongbin Zhang (i) ${ }^{1 凶}$

Sarpagine-Ajmaline-Koumine type monoterpenoid indole alkaloids represent a fascinating class of natural products with polycyclic and cage-like structures, interesting biological activities, and related biosynthetic origins. Herein we report a unified approach towards the asymmetric synthesis of these three types of alkaloids, leading to a collective synthesis of 14 natural alkaloids. Among them, akuammidine, 19-Z-akuammidine, vincamedine, vincarine, quebrachidine, vincamajine, alstiphylianine J, and dihydrokoumine are accomplished for the first time. Features of our synthesis are a new Mannich-type cyclization to construct the key indole-fused azabicyclo[3.3.1]nonane common intermediate, a $\mathrm{Sml}_{2}$ mediated coupling to fuse the aza-bridged E-ring, stereoselective olefinations to install either the 19-E or 19-Z terminal alkenes presented in the natural alkaloids, and an efficient iodo-induced cyclization to establish the two vicinal all-carbon quaternary centers in the Koumine-type alkaloids.

\footnotetext{
${ }^{1}$ Key Laboratory of Medicinal Chemistry for Natural Resource, Ministry of Education; Yunnan Provincial Center for Research \& Development of Natural Products; School of Chemical Science and Technology, Yunnan University, Kunming 650091, P. R. China. email: xdyang@ynu.edu.cn; zhanghb@ynu.edu.cn
} 
T he sarpagine alkaloids feature a polycyclic ring system with an azabicyclo[3.3.1]-nonane core. These alkaloids serve as the biogenetic precursors for the more complex ajmaline and koumine type indole alkaloids ${ }^{1-3}$. The sarpagine-ajmalinekoumine type alkaloids are among the most important group of monoterpenoid indole alkaloids ${ }^{4-6}$. Those biogenetically related alkaloids have been isolated mainly from the medicinal plant family Apocynaceae and Loganiaceae, especially from the genera Alstonia, Rauwolfia, and Gelsemium in low natural abundance $^{7-10}$. A number of these alkaloids possess important biological activities, including anti-leishmanial ${ }^{11}$, anti-malaria parasites $^{12}$, anti-inflammatory ${ }^{13}$, antihypertensive ${ }^{14}$, anticancer ${ }^{15}$, and accelerating the sciatic nerve regeneration activities ${ }^{16}$. The typical molecules are indicated in Fig. 1. Because of their characteristic indole-fused azabicyclo[3.3.1]-nonane structures and prominent biological activities, sarpagine-ajmaline-koumine related alkaloids have attracted attention from the organic synthetic community for decades ${ }^{17-25}$. Synthetic efforts have resulted in a number of elegant strategies and culminated with the synthesis of a series of sarpagine-ajmaline-koumine type alkaloids. Formation of the indole fused azabicyclo[3.3.1]nonane structures could be roughly classified to five categories: the PictetSpengler cyclization (forming the $\mathrm{C} 2-\mathrm{C} 3$ bond) approaches, the intramolecular condensation/addition methodologies (constructing the C15-C16 bond), the transition metal-mediated cyclizations, the cycloaddition/annulation strategies, and the FriedelCrafts acylation approach (forming the C6-C7 bond). The first category includes Tamelen's ${ }^{26}$, Masamune's $s^{27}$, Craig's ${ }^{28}$, Sudhakar's ${ }^{29}$ Pictet-Spengler cyclization procedures, and most recently, Qi's aza-Achmatowicz rearrangement of indole-tethered furan followed by Pictet-Spengler cyclization ${ }^{30}$. The second category constitutes Cook's approach based on Dieckman condensation of carboline derivatives ${ }^{31-42}$, and Bailey's intramolecular Michael addition procedures ${ }^{43,44}$. The third category comprises Martin's approach via Pauson-Khand reaction ${ }^{45}$, olefin metathesis ${ }^{46}$, Kuethe's intramolecular Heck cyclization approach ${ }^{47}$, and most recently, Zhang's Copper-catalyzed oxidative cyclization ${ }^{48,49}$. The fourth category includes Ohba's intramolecular oxazole-olefin Diels-Alder cycloaddition ${ }^{50}$, and Gaich's $[5+2]$ cycloaddition followed by ring enlargement and Fischer indole annulation ${ }^{51-53}$. The last category is Kwon's intramolecular Friedel-Crafts acylation approach ${ }^{54}$. Numerous sarpagine-ajmaline-koumine type alkaloids have been synthesized based on above-mentioned strategies, however, quebrachidine, vincamedine, and its analogues are not yet conquered to our knowledge by total synthesis ${ }^{17-25}$. The natural alkaloid quebrachidine (5) and its $N$-methyl congener vincamajine (6) and vincamedine (7) are among the most highly functionalized and synthetically challenging ajmaline type alkaloids. Quebrachidine is the biogenetic precursor of bisindole alkaloid alstonisidine (15) and has been isolated for more than 50 years ${ }^{55}$, while vincamedine and alstiphyllanines (Fig. 1, 11 and 12) possess potent vasorelaxant activity ${ }^{56}$. It is also noteworthy that alstiphyllanines are 19-Z-derivatives of quebrachidine.

In this work, we report a structure-unit-oriented strategy towards the synthesis of 14 natural monoterpenoid indole alkaloids. Although numerous synthetic methods have been developed for sarpagine-ajmaline alkaloids as well as structurally

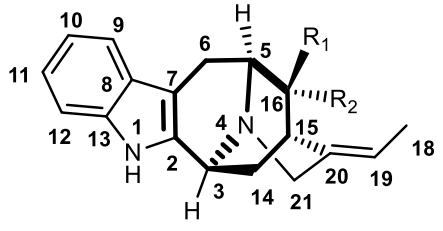

1: $\mathrm{R}_{1}=\mathrm{CO}_{2} \mathrm{Me}, \mathrm{R}_{2}=\mathrm{CH}_{2} \mathrm{OH}$, Akuammidine

2: $\mathrm{R}_{1}=\mathrm{CH}_{2} \mathrm{OH}, \mathrm{R}_{2}=\mathrm{COOMe}$, Polyneuridine

3: $R_{1}=\mathrm{CHO}, \mathrm{R}_{2}=$ COOMe, Polyneuridine aldehyde
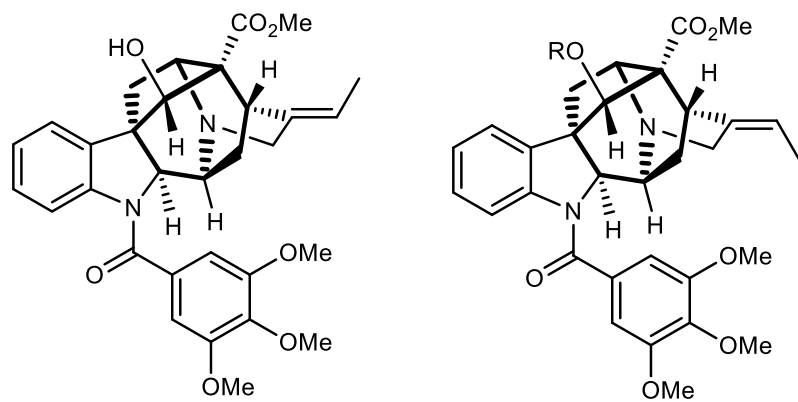

10: 1-N-(3',4',5'-Trimethoxybenzoyl)-vincamajine
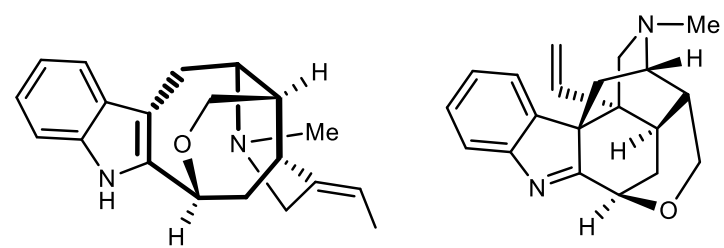

13: 19-(Z)-Taberpsychine

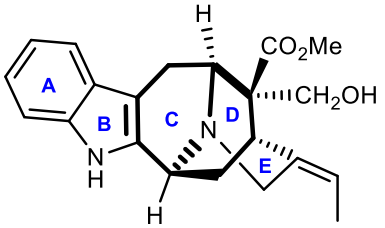

4: 19-(Z)-akuammidine

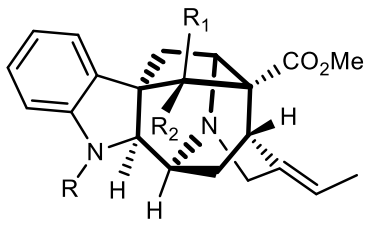

5: $R=R_{\mathbf{2}}=H, R_{1}=O H$, Quebrachidine

6: $R=M e, R_{1}=O H, R_{2}=H$, Vincamajine

7: $R=M e, R_{1}=O A c, R_{2}=H$, Vincamedine

8: $R=R_{1}=H, R_{2}=O H$, Vincarine

9: $R=M e, R_{1}=H, R_{2}=O H$, Vincamajinine
11: $\mathbf{R}=$ Ac, Alstiphyllanine $\mathbf{J}$ 12: $\mathbf{R}=\mathrm{H}$, Alstiphyllanine $M$

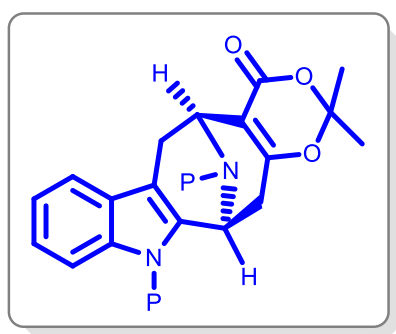

16: common intermediate for Sapargine, ajmaline and koumine type alkaloids

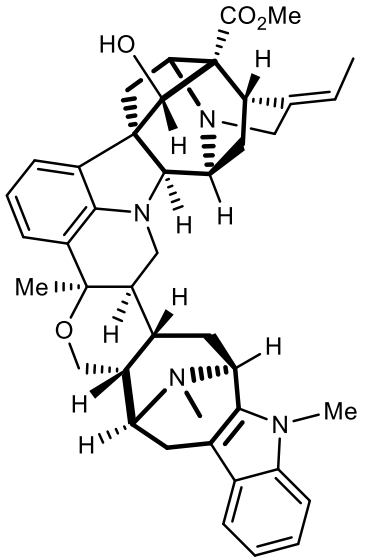

15: Alstonisidine

Fig. 1 Some representative sarpagine-ajmaline-koumine type alkaloids. In this work, alkaloids 1-9, 11, 13, and 14 are synthesized. 


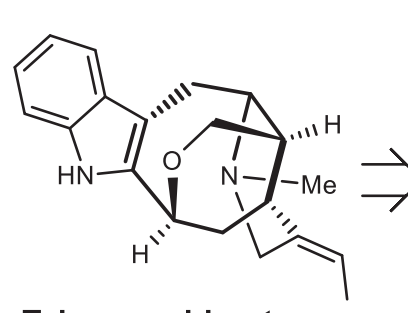

Taberpsychine type
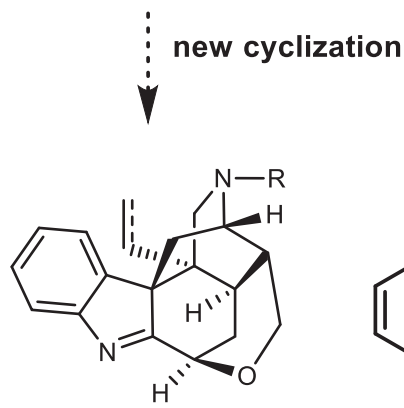

Koumine type

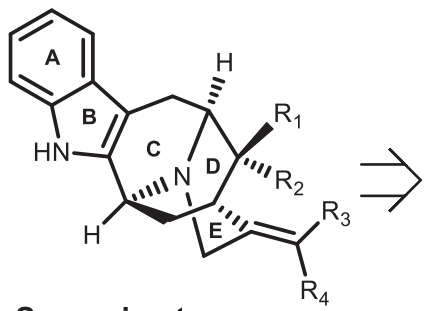

Sarpagine type
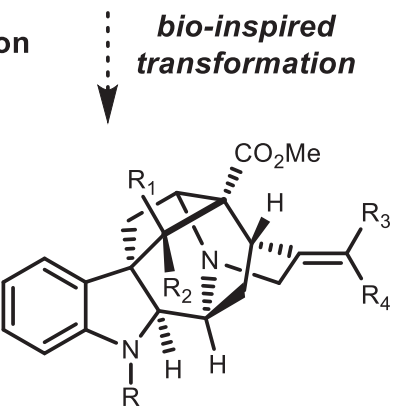

Ajamaline type

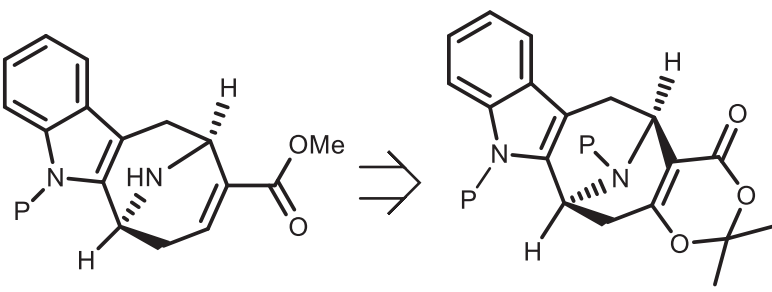

20

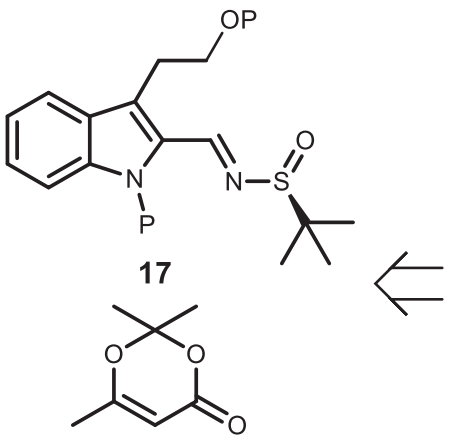

18
16: common intermediate

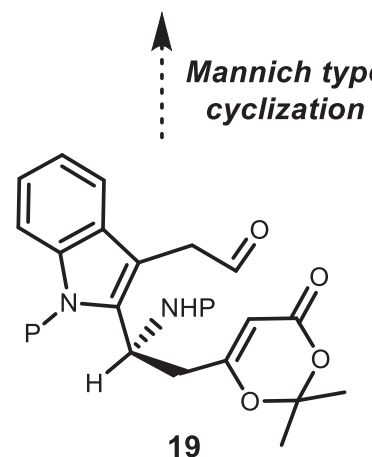

Fig. 2 Retrosynthetic analysis based on amide-mediated Mannich-type cyclizations. The collective synthesis of sarpagine-ajmaline-koumine type alkaloids are based on common intermediate $\mathbf{1 6 .}$

related analogues, flexible and unified synthetic routes that lead to collective synthesis of these types of alkaloids, especially towards both $19-E$ and $19-Z$ isomers, are rarely documented ${ }^{17-25}$.

\section{Results}

Retrosynthetic analysis. As part of our ongoing program in seeking flexible and divergent synthetic strategies toward bioactive natural products and its analogues ${ }^{57-61}$, we recently disclosed a Lewis acid-mediated intramolecular Prins reaction of dioxinones to construct medium-sized carbocycles bearing oxabridged bicyclic structural units ${ }^{62}$. We envisioned that the Mannich-type-cyclization version of our previous protocol might lead to the highly functional 9-azabicyclo[3.3.1]nonane ring system (16, Fig. 2), an ideal intermediate with properly located functional groups and necessary carbons for the synthesis of akuammidine-related alkaloids shown in Fig. 1.

Our retrosynthetic analysis is outlined in Fig. 2. One of the key transformations in the synthetic plan is cascade cyclizations (Fig. 2, transformation of $19 \rightarrow \mathbf{1 6}$ ) to form the aza-bridged eightmembered ring system from indole fused chain compound (19). Although we have succeeded previously the synthesis of oxabridged medium-sized ring systems ${ }^{62}$, and aza-Prins cyclization have been used elegantly in the synthesis of six-membered carbocyclic rings ${ }^{63,64}$, the proposed tandem cyclizations to obtain aza-bridged bicyclo[3.3.1] nonane common structure units (16) are challenging. We postulated that an amine or amide participated intramolecular Mannich-type reaction of dioxinones under Lewis acid conditions would provide access to the required common intermediate (16, Fig. 2). Based on the well-located functional groups, with proper manipulations, we would be able to synthesize the natural alkaloids shown in Fig. 1.

Preparation of the common azabicyclo[3.3.1]-nonane core. We commenced our synthetic studies with the preparation of tertbutanesulfinamide 19a (Fig. 3). The desired sulfinamide 19a was obtained after two steps in 63\% yield from known aldehyde 17a, obtained two steps from commercially available tryptophol ${ }^{65}$. Our initial plans were oxidation of sulfinamide 19b (after removal of PMB with DDQ) or amine 19c (after removal of PMB with DDQ and the tert-butanesulfinyl group with iodine ${ }^{61}$ ) to its corresponding aldehydes and subjected it to the proposed Mannich-type reactions. Unfortunately, both substrates (19b and 19c) failed to yield reasonable amounts of aldehydes under a number of oxidation conditions. To circumvent this problem, the amine was converted to amide 19d with Boc-anhydride $(71 \%$ overall yields in three steps from 19a). Oxidation of alcohol 19d with IBX ${ }^{66}$ provided an unstable aldehyde (19e), which was used immediately in the cyclization under Lewis acid conditions. To our delight, the cascade sequence proceeded precisely and produced the desired 16a as a single diastereoisomer in 57\% yield (gram-scale) over two consecutive steps. The correct stereochemistry of the pentacyclic core was confirmed by single crystal $\mathrm{X}$-ray analysis.

With the common intermediate 16a in hand, we next explored the synthesis of sarpagine-ajmaline alkaloids indicated in Fig. 1, aiming to synthesize both $19-E$ and $19-Z$ natural isomers. Treatment of 16a with sodium methoxide afforded methyl ester 20a in $94 \%$ yield. Deoxygenation of enol 20a via formation of triflate with Comins reagent followed by palladium-catalyzed hydrogenolysis ${ }^{67,68}$ provided 20 b in excellent yield. Next, the Boc protecting group was removed with trifluoroacetic acid in dichloromethane and the resultant was treated with $(Z)-1$ bromo-2-iodobute-2-ene to afford intermediate 20c. Our initial plan to fuse the $\mathrm{C}_{15}-\mathrm{C}_{20}$ carbon-carbon bond of akuammidinerelated alkaloids was to follow the well-established procedure via reductive Heck-type reactions ${ }^{69-76}$. To our dismay, no desired product was obtained under various palladium and nickel mediated conditions, with dehalogenation product being isolated in most cases (see Supplementary Information for details). Treatment of iodide 20c with tri- $n$-butyltin hydride ${ }^{77}$ was also fruitless, with a complex mixture being obtained. Difficulty in assembling the bridged E-ring prompted us to explore alternative methods. It was of our interests to use Skrydstrup's chemistry ${ }^{78-84}$, namely $\mathrm{SmI}_{2}$ mediated acyl radical reaction. As indicated in Fig. 4, Skrydstrup coupling would lead to a tempting product, namely intermediate $\mathbf{2 3}$, which bears a tunable carbonyl moiety for further manipulation towards desired $19-E$ and $19-Z$ 
<smiles>CC(C)(C)OCCc1c(C=O)n(C(C)(C)C)c2ccccc12</smiles>

$17 a$

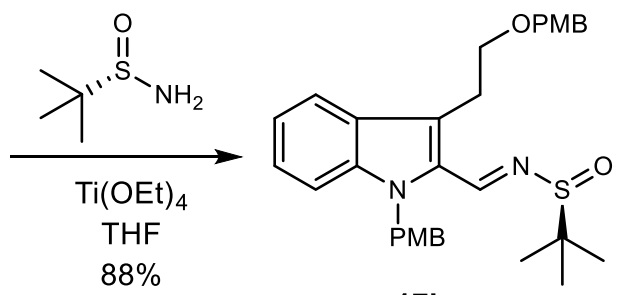

$17 \mathrm{~b}$

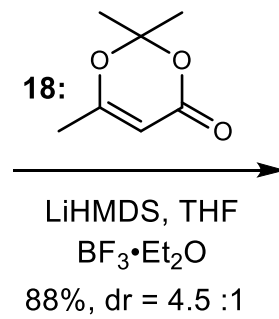

$88 \%, d r=4.5: 1$

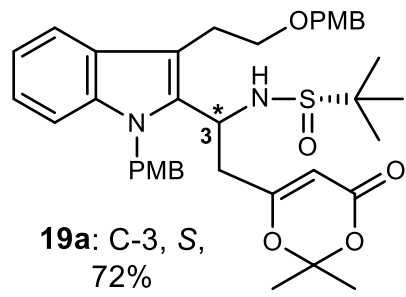

19a': C-3, $R$, $16 \%$

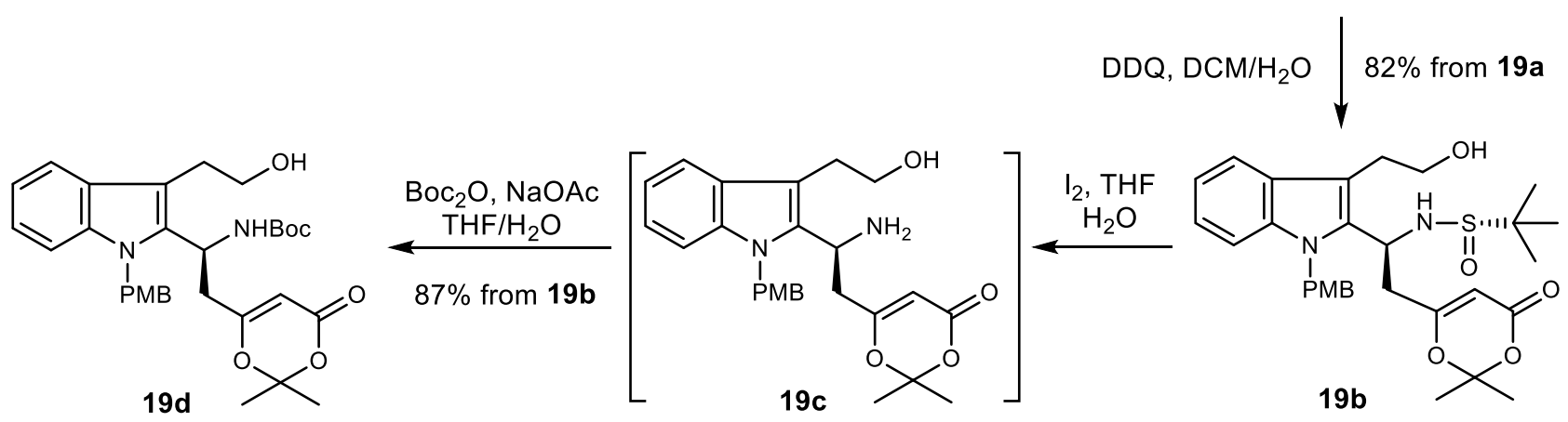

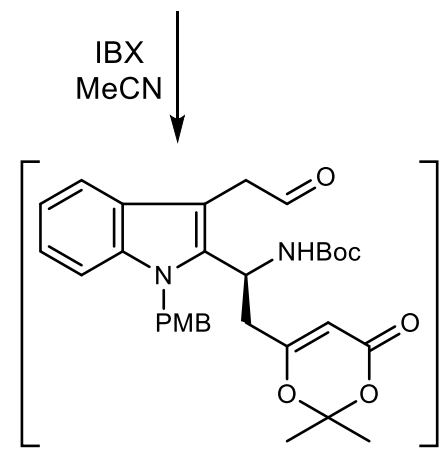

$19 e$

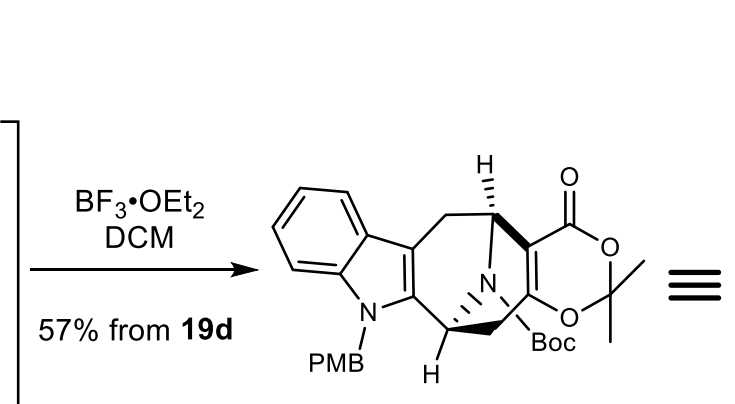

$16 a$

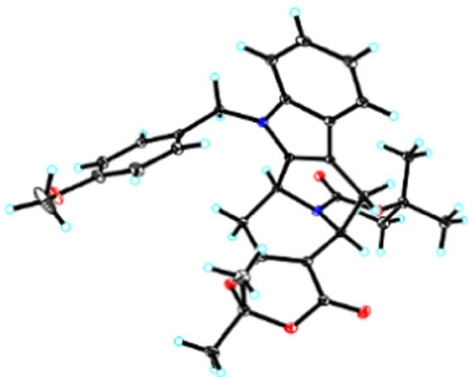

crystal structure of $\mathbf{1 6 a}$

Fig. 3 Gram-scale synthesis of common intermediate 16a. The key transformations to access the common intermediate 16a include a vinylogous Mannich reaction of lithium dienolate $\mathbf{1 8}$, and a Lewis acid mediated Mannich type cyclization of 19e. Ac acetyl, Boc $t$-butoxycarbonyl, DCM dichloromethane, DDQ 2,3-dichloro-5,6-dicyano-1,4-benzoquinone, IBX o-iodoxybenzoic acid, LiHMDS lithium bis(trimethylsilyl)amide, PMB p-methoxybenzyl, THF tetrahydrofuran.

olefins. The intermediate $\mathbf{2 0 b}$ was then transformed to its corresponding $\mathrm{N}$-acyl-oxazolidinone derivative 22 (2 steps, $76 \%)$. The well-established Skrydstrup's conditions $\left(\mathrm{SmI}_{2} / \mathrm{H}_{2} \mathrm{O} /\right.$ THF) unfortunately failed to promote the desire cyclization, however, after examining several other conditions, we finally established a reproducible procedure for constructing the bridged

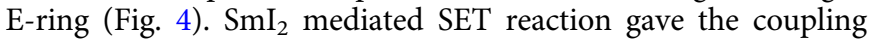
product in $76 \%$ yield with excellent diastereoselectivity (single $\mathrm{C}_{16}$ isomers). We deduced that the chelation of samarium diiodide with the carbonyl groups as proposed by Skrydstrup ${ }^{79}$ (Fig. 4) secured the desired cyclization. The NOESY correlations of $\mathrm{H}-16$ to both $\mathrm{H}-14$ and $\mathrm{H}-15$ for compound 23 indicated a $\beta$ orientation of the H-16 (Fig. 4, see Supplementary Information for spectra). Thus, the pentacyclic skeletons for akuammidinerelated alkaloids had been successfully assembled and ready for late-stage manipulation.

We next focused on the selective synthesis of $E$ and $Z$ alkenes with ketone 23. Treatment of ketone 23 with Wittig reagent ${ }^{85-87}$ in THF in the presence of NaHMDS provided olefins $\mathbf{2 4}$ (isolated as the major isomer, $83 \%$ yield) and $\mathbf{2 5}$ (15\% yield) in a $98 \%$ combined yield. Treatment of ketone 23 with Julia reagent ${ }^{88}$ in THF altered the ratio and provided olefin $\mathbf{2 5}$ as the major isomer in $80 \%$ yield together with $\mathbf{2 4}$ (19\% yield). Suitable crystal was obtained from compound $\mathbf{2 5}$, and the $19-Z$ stereochemistry was established by X-ray crystallography (Fig. 4). This new approach enabled access to either 19-E (Wittig-olefination, trans-selective) or 19-Z (Julia-olefination, cis-selective) isomers selectively.

Total synthesis of natural sarpagine-type and ajmaline-type alkaloids. With the advanced intermediates (24 and 25) in hand, we next began the journey towards akuammidine-related alkaloids. Treatment of $\mathbf{2 4}$ with LDA followed by formaldehyde (Fig. 5), we obtained desired C16 stereoisomer 26 (55\% yield) together with byproduct 27 ( $17 \%$ yield). Using compound 25, similar operation provided intermediates 28 (42\%) and $29(30 \%)$. It is worthwhile to note that the undesired C-16 isomers could be recycled, a retro-Aldol reaction in the presence of sodium hydride converted 27 and $\mathbf{2 9}$ back to its corresponding starting materials (24: 86\% yield, and 25: 83\% yield, Fig. 5). Deprotections of PMB in intermediates 26 and 28 under acidic conditions furnished the first total synthesis of natural akuammidine (1, confirmed by $\mathrm{X}$-ray analysis) and 19-Z-akuammidine (4). The NMR spectra as well as physical data of our synthetic sample were consistent with those reported in the literature 89,90 .

Having secured the total synthesis of akuammidines, we next turned our attention to the synthesis of more challenging 

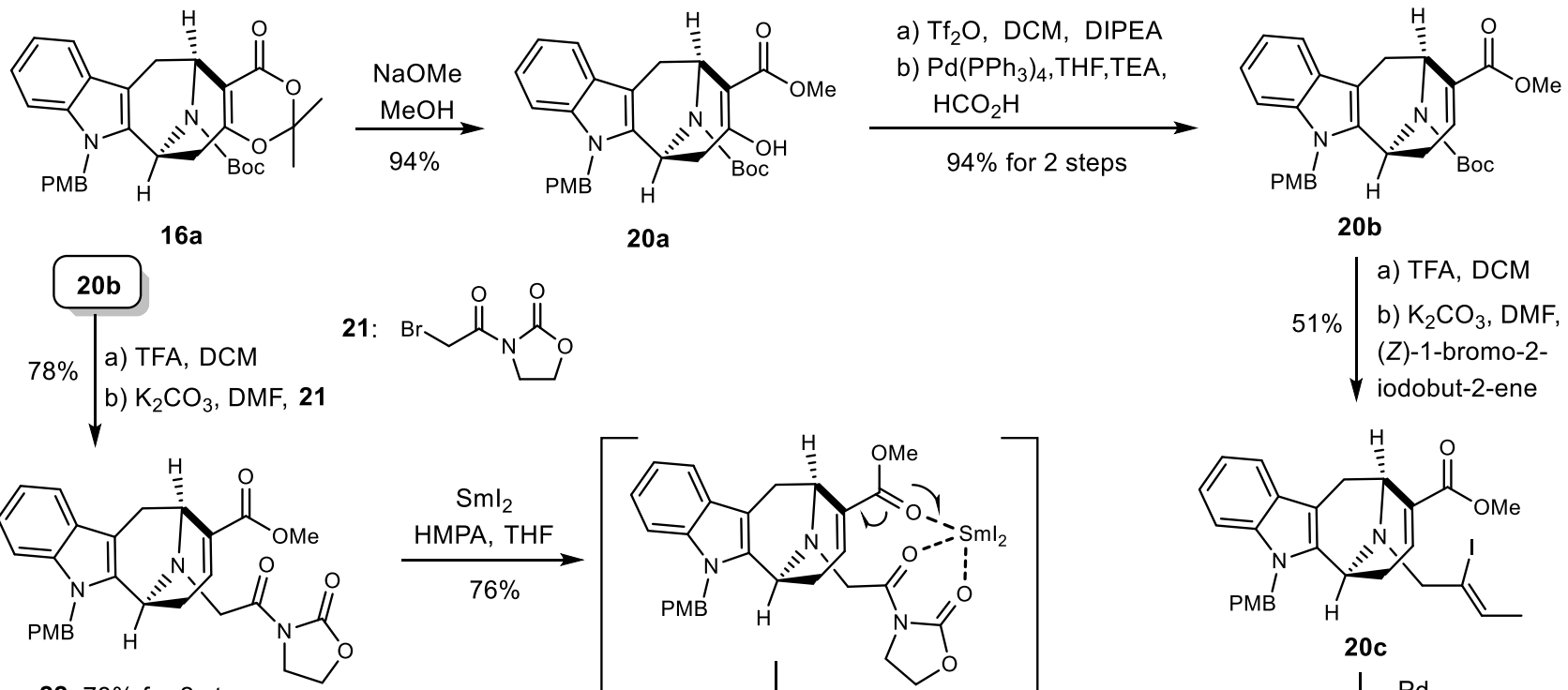

21:

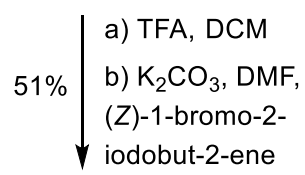

22: $78 \%$ for 2 steps
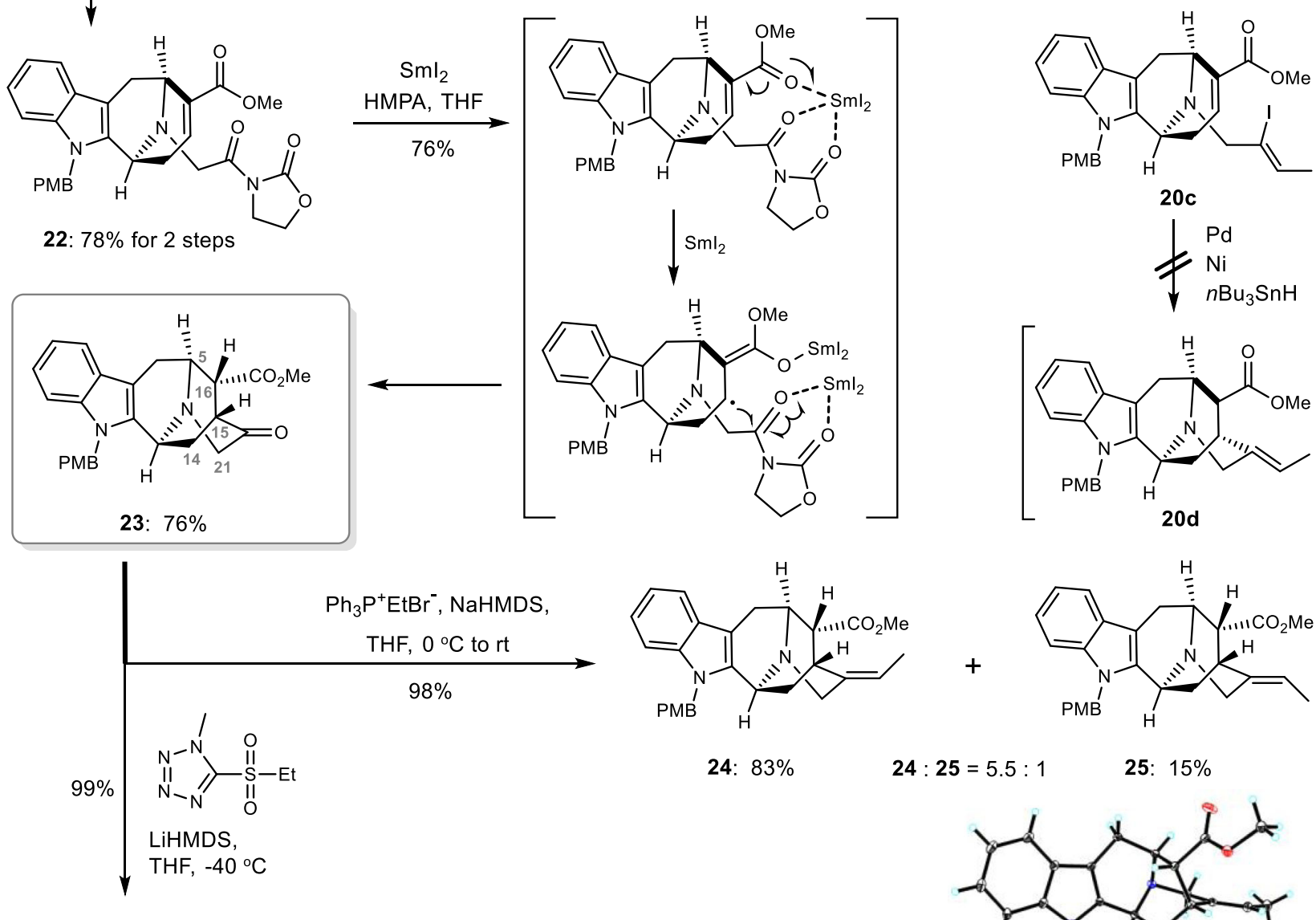

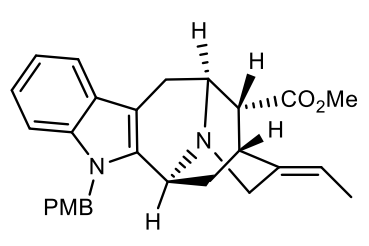

25: $80 \%$

$25: \mathbf{2 4}=4.2: 1$

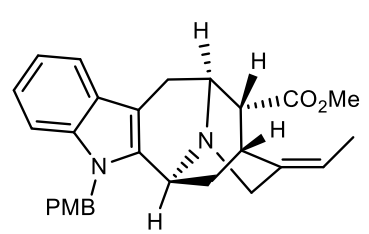

24: $19 \%$

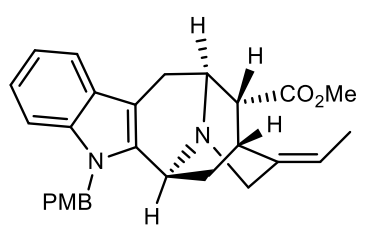

24: $83 \%$

$24: \mathbf{2 5}=5.5: 1$

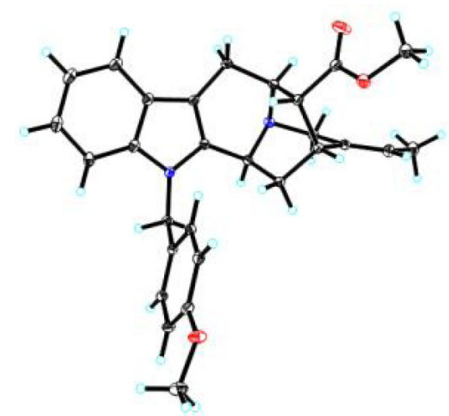

crystal structure of $\mathbf{2 5}$

Fig. 4 Synthesis of the common sarpagine type skeletons. After construction of the bridged E-ring via Sml 2 mediated SET reaction, the double bonds were assembled by Wittig reaction (E-selectivity) and Julia olefination (Z-selectivity). DIPEA diisopropylethylamine, DMF N,N-dimethylformamide, HMPA hexamethylphosphoramide, Me methyl, Ph phenyl, TEA triethylamine, Tf trifluoromethanesulfonyl, TFA trifluoroacetic acid, Et ethyl, NaHMDS sodium bis(trimethylsilyl)amide.

ajmaline-type alkaloids. To increase the ratio of intermediate 27 , a number of additives were used including $\mathrm{BF}_{3} \cdot \mathrm{Et}_{2} \mathrm{O}, \mathrm{TMSCl}$ (trimethylsilyl chloride), TMEDA ( $N, N, N^{\prime}, N^{\prime}$-tetramethylethylenediamine), and HMPA. The addition of HMPA could slightly increase the ratio of compound 27 (30\% yield) while undesired C-16 isomer 26 was converted back to starting material (24) through retro-aldol reaction in $84 \%$ yield (Fig. 6). Treatment of 27 with TFA in the presence of $\mathrm{PhSH}$ yielded natural polyneuridine (2) in $90 \%$ yield ${ }^{91,92}$. Corey-Kim oxidation of 2 provided the biosynthetic precursor polyneuridine aldehyde $(3)^{3,4,21}$. Upon exposure to acidic conditions $\left(\mathrm{Ac}_{2} \mathrm{O}\right.$ and $\mathrm{TfOH}$, modification of Cook's conditions ${ }^{35}$ ), an inseparable mixture of C17 stereoisomers (30a and 30b, 63\% yields in two steps, $d r=1.1: 1$ based on $\left.{ }^{1} \mathrm{H}-\mathrm{NMR}\right)$ was obtained. Reduction of the mixture with sodium cyanoborohydride gave amines $\mathbf{3 1 a}$ and $\mathbf{3 1 b}$ (inseparable mixture) in $87 \%$ combined yield (Fig. 6). We 


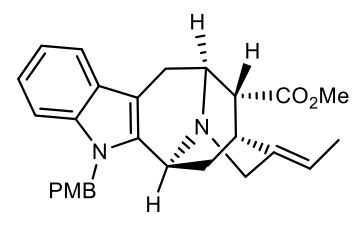

24

$$
\begin{array}{c|l}
72 \% & \text { LDA, }\left(\mathrm{CH}_{2} \mathrm{O}\right)_{n}, \\
3.2: 1 & \text { THF, } 0{ }^{\circ} \mathrm{C} \text { to rt }
\end{array}
$$
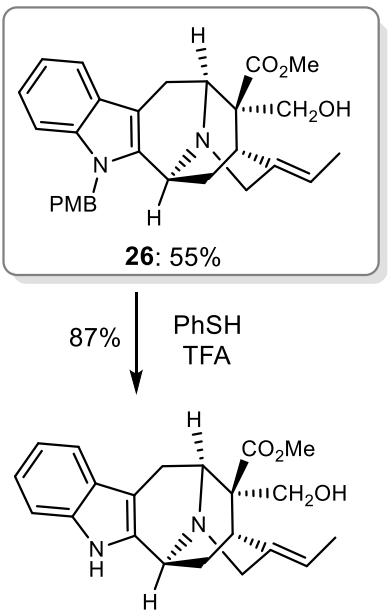

1: Akuammidine
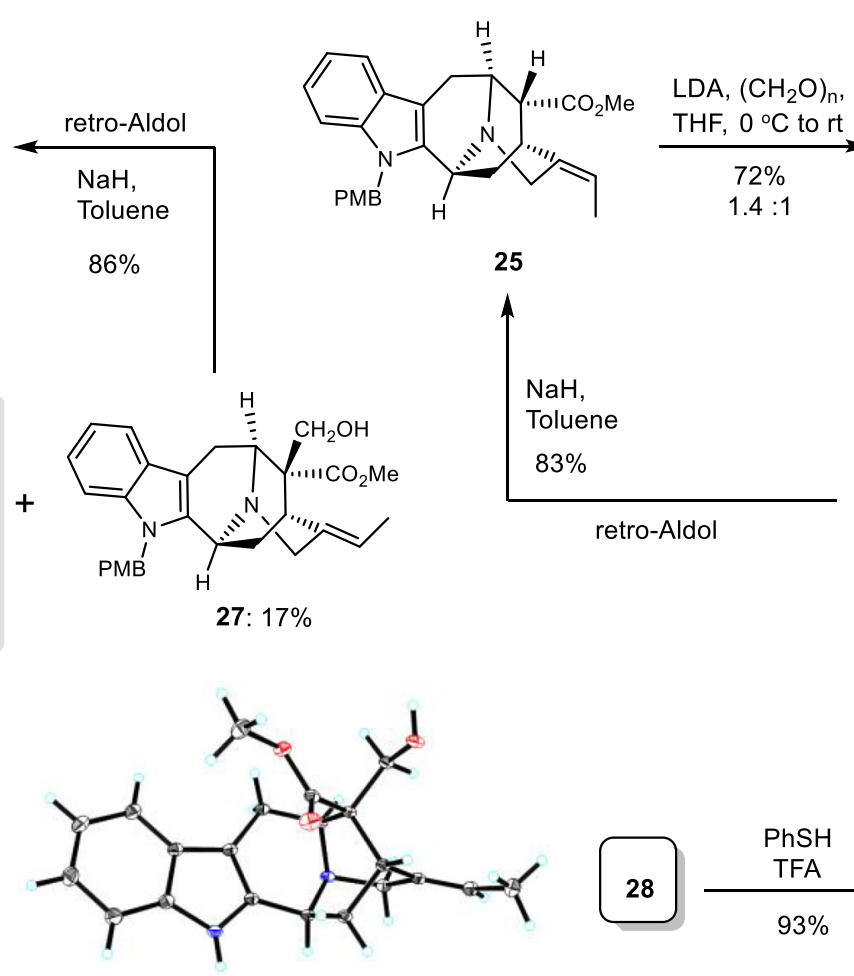

crystal structure of 1
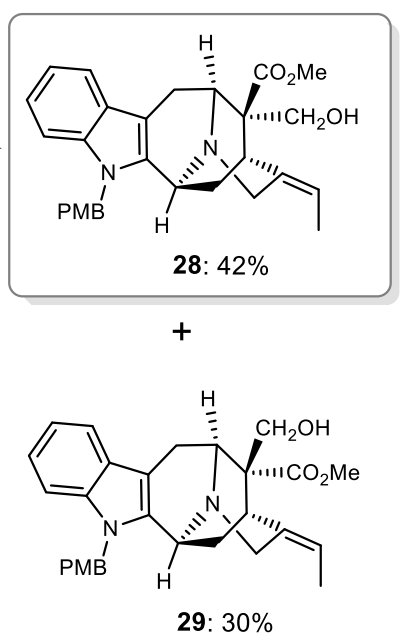

29: $30 \%$

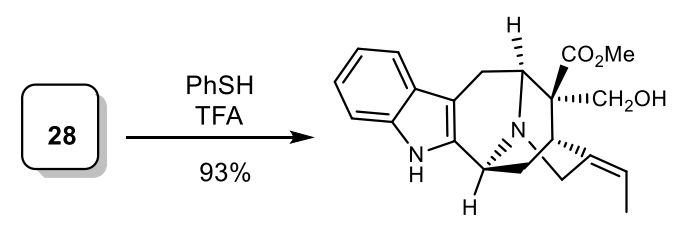

4: 19-(Z)-akuammidine

Fig. 5 Synthesis of akuammidine (1) and 19-Z-akuammidine (4). Aldol condensation with formaldehyde. LDA lithium diisopropylamide.

envisioned that the inseparable $\mathrm{C} 17$-acetate isomers might react differently under hydrolysis conditions due to its different steric hindrances. Thus, the mixture of 31a and 31b were treated with $\mathrm{K}_{2} \mathrm{CO}_{3}$ in methanol. This process provided unchanged acetate 31 a in $50 \%$ yield together with natural vincarine $(8,47 \%$ yield, from hydrolysis of $\mathbf{3 1 b}$, the first total synthesis), the diastereoisomer of quebrachidine ${ }^{6,36,93}$. Reductive amination of 31a with formaldehyde furnished the first total synthesis of vincamedine $(7)^{94}$. Hydrolysis of 7 afforded vincamajine $(\mathbf{6})^{94}$ in $93 \%$ yield. Further hydrolysis of 31a provided the first total synthesis of natural alkaloid quebrachidine $(5)^{55,95}$. The structure of synthetic quebrachidine was confirmed by X-ray crystallography.

Treatment of vincarine (8) with sodium cyanoborohydride in the presence of formaldehyde gave vincamajinine $(\mathbf{9})^{36}$. The NMR spectra and physical data of our synthetic samples were in complete agreement with the reported data. It is noteworthy that vincarine has been documented in literature for more than 50 years, no complete reported NMR data are available in the literature ${ }^{6,36}$.

Next, we concentrated on the total synthesis of alstiphyllanines (with a 19-Z-olefin moiety), a number of bioactive ajmaline-type alkaloids differs from each other only in amide substituents ${ }^{56}$. Aldol reaction of compound 25 (from Julia-olefination in Fig. 4) in the presence of HMPA afforded desired isomer 29 in $37 \%$ yield (Fig. 7). Treatment of 29 with TFA in the presence of $\mathrm{PhSH}$ afforded amine 32 in $86 \%$ yield. Oxidation of 32 under CoreyKim conditions gave aldehyde 33, which was directly subjected to acidic conditions to yield cyclization products $34 \mathbf{a}$ and $\mathbf{3 4 \mathbf { b }}(60 \%$ yield over two steps, $\sim 1: 1.45$ ratio) as an inseparable mixture of C17 diastereoisomers. Selective reduction of the imine moiety presented in 34a and $\mathbf{3 4} \mathbf{b}$ with sodium cyanoborohydride afforded amines 35a and 35b (inseparable mixture of $\mathrm{C} 17$ diastereoisomers) in $90 \%$ combined yield. Hydrolysis of the mixture of $\mathbf{3 5 a}$ and $\mathbf{3 5 b}$ under our standard conditions provided separable alcohol 36 (59\%) and acetate $\mathbf{3 5 a}$ (37\%). The alcohol 36 could be recycled by oxidation with $\mathrm{MnO}_{2}$ in dichloromethane ${ }^{96}$. Finally, treatment of 35a with eudesmoyl chloride in pyridine furnished the first total synthesis of alstiphyllanine J (11). The NMR spectra of our synthetic sample agree well with the reported data $^{56}$.

Total synthesis of natural koumine-type alkaloids. Finally, to further demonstrate the flexibility of our strategy, we decided to synthesize the challenging koumine-type alkaloids, a cage-like alkaloid family with two vicinal all-carbon quaternary centers ${ }^{5}$. There are two key issues in the synthesis of koumine-type alkaloids from common intermediate $\mathbf{2 5}$, namely the epimerization of the $\mathrm{C}-16$ stereochemistry and a cyclization to form the two vicinal all-carbon quaternary centers. Experiments (see Supplementary Information) to epimerize the C-16 stereochemistry using a number of bases and proton donors failed to produce any desired epimer 25a (Fig. 8). Next, intermediate 25 was converted to iodide $25 \mathbf{b}$, aiming to alter the C-16 stereochemistry via radical reduction. Treatment of compound $25 \mathbf{b}$ with $n$ - $\mathrm{Bu}_{3} \mathrm{SnH}$ failed again to provide 25a. Light-induced radical reductions were then attempted ${ }^{97}$. After extensive experiments (see Supplementary Information for details), we finally found that reduction of $\mathbf{2 5 b}$ with catalytic amount of $\left[\operatorname{Ir}(\mathrm{ppy})_{2}(\mathrm{dtbbpy})\right] \mathrm{PF}_{6}$ in the presence of DIPEA and (TMS $)_{2} \mathrm{NH}$ under blue LED $\left(-60^{\circ} \mathrm{C}\right)$ provided desired $\mathbf{2 5 a}$ in $47 \%$ yield (Fig. 8), with 25 being recovery in 16\% yield. Deprotection of 25a with TFA followed by reduction with $\mathrm{LiAlH}_{4}$ (LAH) gave natural koumidine 25c (80\% yield over two steps $)^{98,99}$.

Treatment of the koumidine $25 \mathrm{c}$ with methyl chloroformate afforded amide 37 in $69 \%$ isolated yield. Reduction of $\mathbf{3 7}$ with LAH provided natural $19-Z$-taberpsychine $(\mathbf{1 3}, \quad 78 \%)^{100}$. Although alkaloid koumine has been prepared by both partial synthesis and total synthesis in the literature ${ }^{5,24,49,99}$, cyclizations 

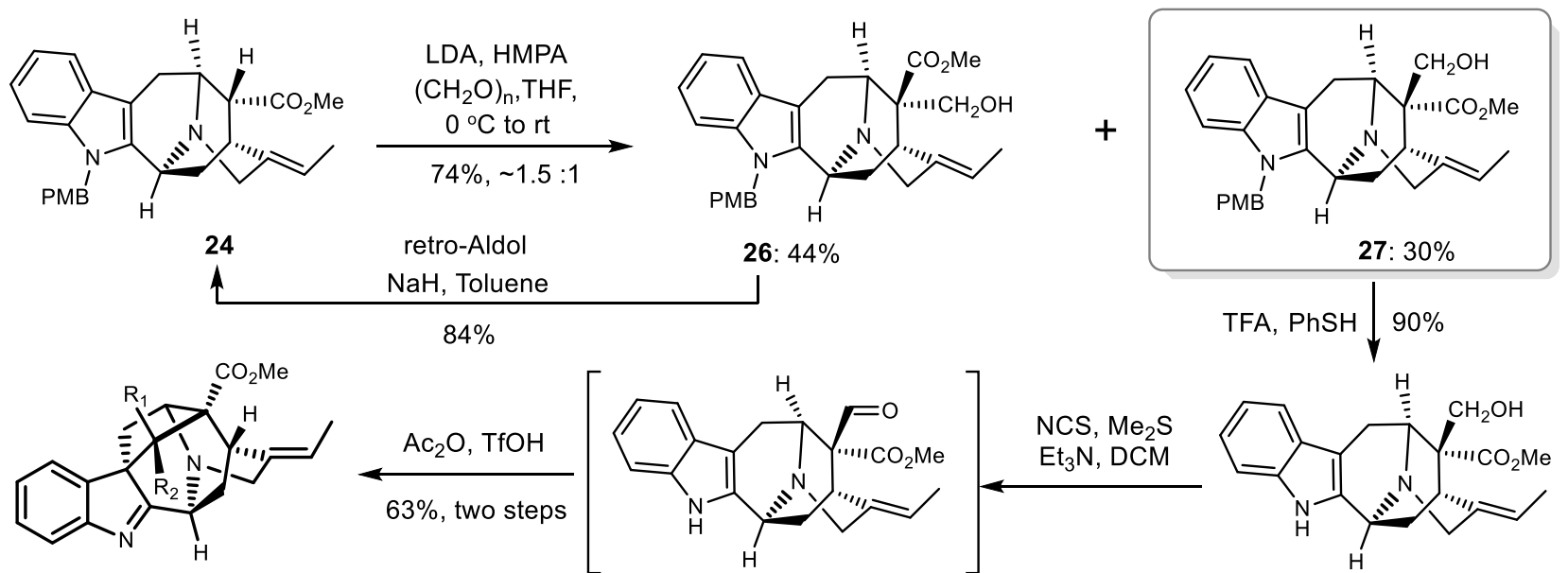

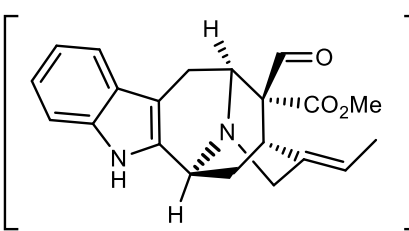

3: Polyneuridine aldehyde

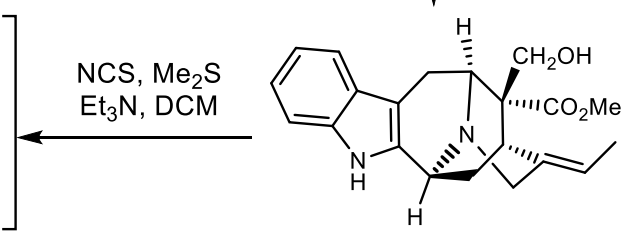

2: Polyneuridine

30a: $R_{1}=O A c, R_{2}=H ; 30 b: R_{2}=O A c, R_{1}=H$ $87 \%\rfloor \mathrm{NaBH}_{3} \mathrm{CN}, \mathrm{AcOH}$

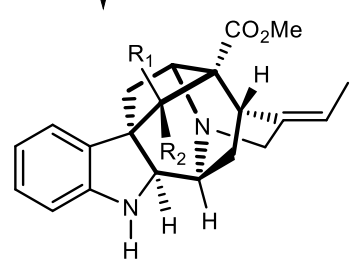

mixture of $31 \mathbf{a}$ and $31 \mathbf{b}, \sim 1.1: 1$

31a: $R_{1}=O A c, R_{2}=H ; 31 b: R_{2}=O A c, R_{1}=H$

$\mathrm{MeOH}, \mathrm{K}_{2} \mathrm{CO}_{3}, 10^{\circ} \mathrm{C}, 4 \mathrm{~h}$

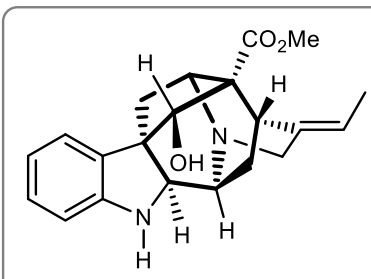

8: Vincarine, $47 \%$

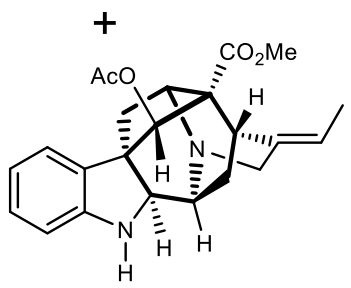

31 a, $50 \%$

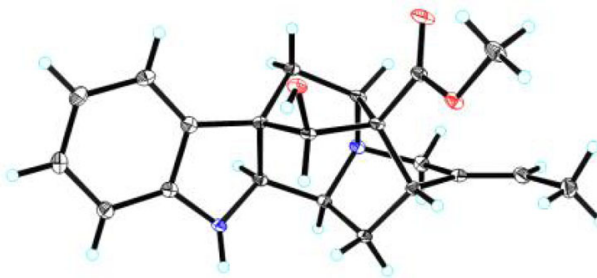

crystal structure of $\mathbf{5}$

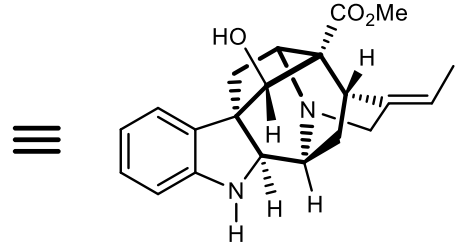

5: Quebrachidine

$85 \%\left\{\begin{array}{l}\mathrm{MeOH}, \mathrm{K}_{2} \mathrm{CO}_{3}, \\ 25^{\circ} \mathrm{C}, 36 \mathrm{~h}\end{array}\right.$

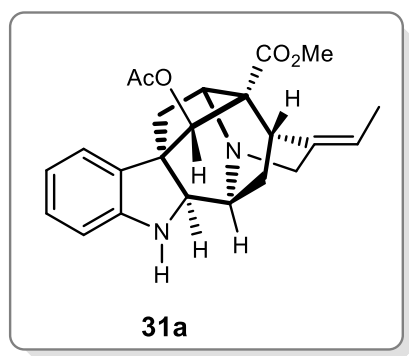

9: Vincamajinine

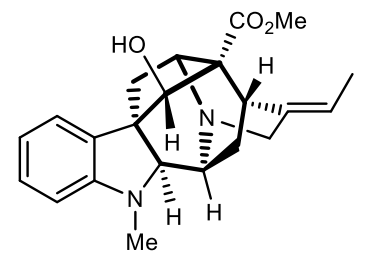

6: Vincamajine

Fig. 6 Synthesis of ajmaline type alkaloids. Quebrachidine, vincarine, vincamedine, and vincamajine were synthesized for the first time. NCS Nchlorosuccinimide.

to construct the vicinal all-carbon quaternary centers in kouminetype structures generally followed the chemistry developed by Liu $\left(\mathrm{SeO}_{2} / \mathrm{H}_{2} \mathrm{O} \text {, oxidation followed by cyclization in low yield }\right)^{101}$ and Sakai $\left(\mathrm{Pd}^{0}, \mathrm{NaH}\right.$ in DMF, based on pre-synthesized 18-hydroxyl derivatives) ${ }^{102}$. Alternative way was reported recently via gold mediated indolyl addition to allene, and the cyclization adduct was converted to koumine in another five steps ${ }^{49}$. In this synthesis, we designed to fuse the two vicinal all-carbon quaternary centers via iodo-induced indolyl cyclization, as the resulting iodide (38) could be converted to olefin via elimination (Fig. 8). The amide 37 was thus treated with NIS in dichloromethane. To our delight, an efficient cyclization occurred, with iodide $\mathbf{3 8}$ being isolated as a single isomer in $88 \%$ yield. Treatment of iodide 38 with silver acetate in acetic acid furnished the olefin (39). After reduction of the amide bond with $\mathrm{LAH}$, the first total synthesis of dihydrokoumine (40) was 

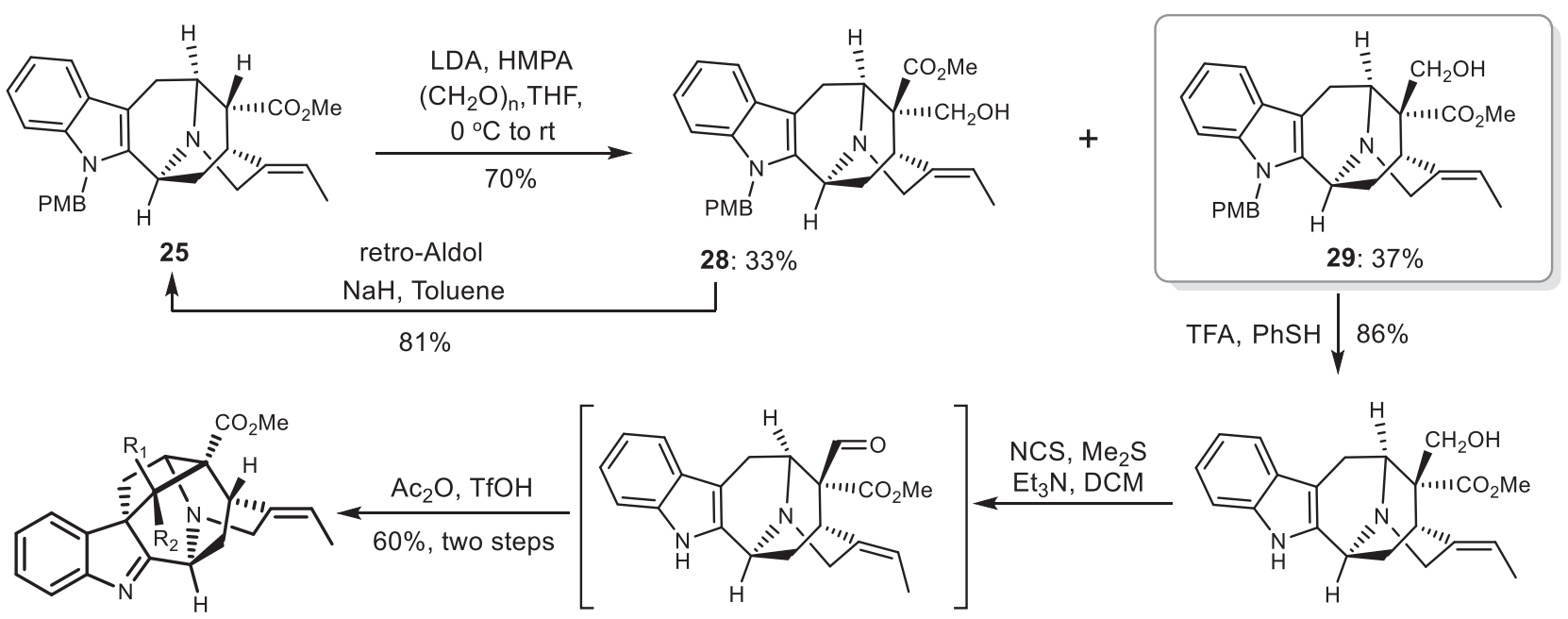

mixture of $34 \mathbf{a}$ and $\mathbf{3 4 b}, \sim 1: 1.45$

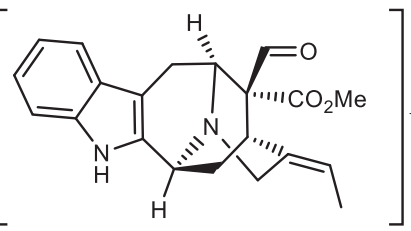

33

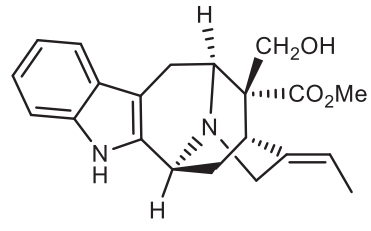

32

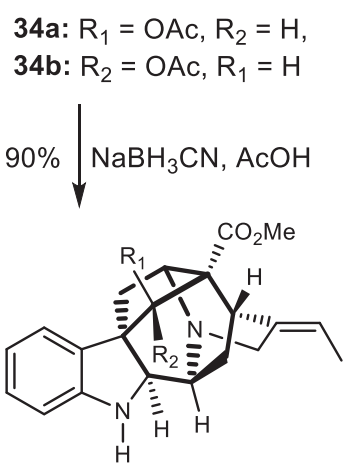

mixture of $35 \mathbf{a}$ and $35 \mathrm{~b}, \sim 1: 1.45$

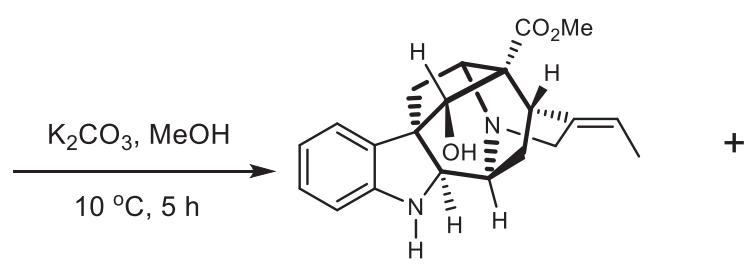

$36,59 \%$

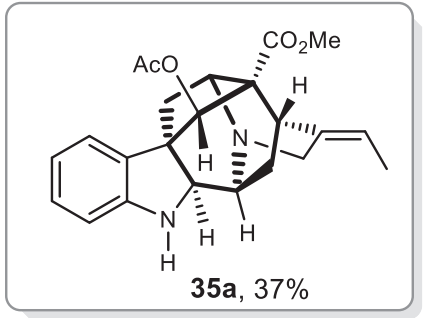

35a: $R_{1}=O A c, R_{2}=H$

35b: $R_{2}=O A c, R_{1}=H$
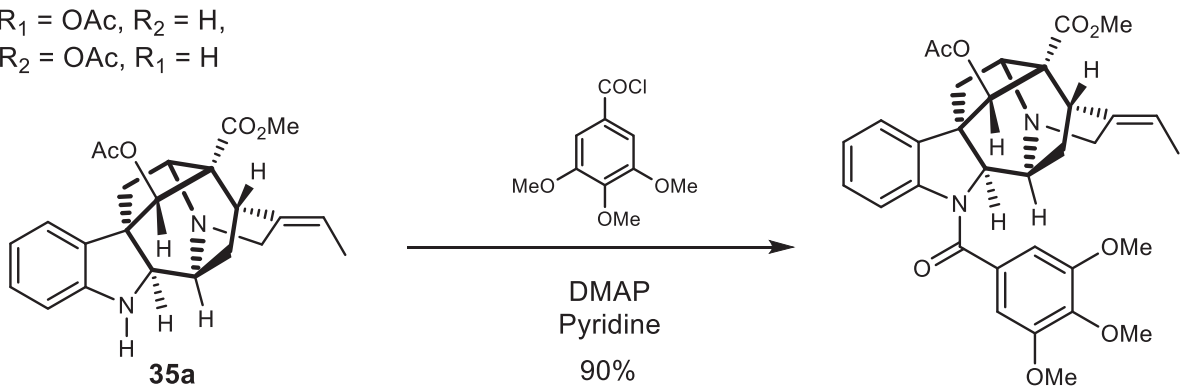

\section{1: Alstiphyllanine $\mathrm{J}$}

Fig. 7 Synthesis of alstiphyllanine J. Alstiphyllanine J has a 19-Z-olefin moiety. DMAP N,N-4-dimethylaminopyridine.

achieved. Oxidation of dihydrokoumine with PhIO in dichloromethane ${ }^{49}$ afforded koumine in $98 \%$ yield. The NMR spectra of our synthetic alkaloids are consistent with those of the natural alkaloids ${ }^{103,104}$.

\section{Discussion}

We have developed a structure unit oriented strategy for the synthesis of cage-like Sarpagine-Ajmaline-Koumine type monoterpenoid indole alkaloids from readily available commercial materials. Among the natural alkaloids synthesized, eight molecules are synthesized for the first time. Key transformations developed are boron trifluoride etherate mediated tandem cascade cyclization to install the azabicyclo[3.3.1]nonane structure unit, samarium(II) iodide mediated SET cyclization to fuse the bridged-hexahydropyridine E-ring, trifluoromethanesulfonic acid induced cyclization to construct the all-carbon quaternary center in ajmaline-type alkaloids, and an efficient iodo-induced cyclization to establish the two vicinal all-carbon quaternary centers in the Koumine-type alkaloids. Our synthetic strategy provided a platform, enabling access to a wide variety of SarpagineAjmaline-Koumine type natural products as well as their analogues, and should be found further application in the synthesis of bisindole alkaloids such as alstonisidine.

\section{Methods}

General. Melting points were measured on a Hanon MP 430 auto melting-point system and are uncorrected. The infrared (IR) spectra were recorded on a Nicolet iS10 FTIR spectrometer with $4 \mathrm{~cm}^{-1}$ resolution and 32 scans between wavenumber of $4000 \mathrm{~cm}^{-1}$ and $400 \mathrm{~cm}^{-1}$. Samples were prepared as $\mathrm{KBr}$ disks with $1 \mathrm{mg}$ of samples in $100 \mathrm{mg}$ of $\mathrm{KBr} .{ }^{1} \mathrm{H}-\mathrm{NMR}$ and ${ }^{13} \mathrm{C}$-NMR spectra were recorded on Bruker Avance 400 and 600 spectrometers. Chemical shifts are reported in parts per million $(\delta)$ referenced to tetramethylsilane $(0.0 \mathrm{ppm})$, chloroform $(7.26 \mathrm{ppm}$ or $77.16 \mathrm{ppm}$ ), and methanol (3.31 ppm or $49.0 \mathrm{ppm})$, respectively. Data for ${ }^{1} \mathrm{H}-\mathrm{NMR}$ and ${ }^{13} \mathrm{C}-\mathrm{NMR}$ spectroscopy are reported as follows: chemical shift $(\delta \mathrm{ppm})$, multiplicity $(\mathrm{s}=$ singlet, $\mathrm{d}=$ doublet, $\mathrm{t}=$ triplet, $\mathrm{q}=$ quartet, $\mathrm{m}=$ multiplet, $\mathrm{br}=$ broad), coupling constant $(\mathrm{Hz})$, integration. High Resolution Mass spectra were taken on AB QSTAR Pulsar mass spectrometer or Aglient LC/MSD TOF mass spectrometer. Optical rotations were recorded on a JASCO P-2000 polarimeter. All 


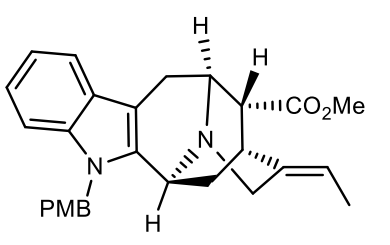

25
LDA, I 2 ,THF, $76 \%$

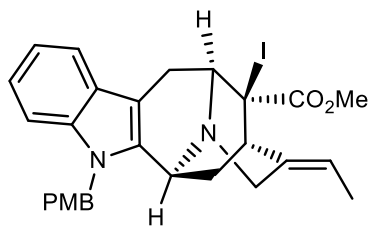

25b

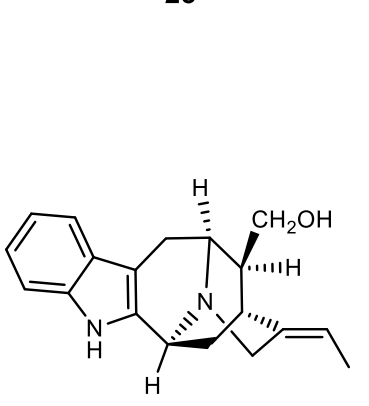

25c: koumidine
1) TFA, Anisole

2) $\mathrm{LiAlH}_{4}, \mathrm{THF}$ $80 \%$

$69 \% \downarrow \begin{aligned} & \mathrm{ClCO}_{2} \mathrm{Me} \\ & \mathrm{THF}^{\mathrm{H}} \mathrm{H}_{2} \mathrm{O}\end{aligned}$

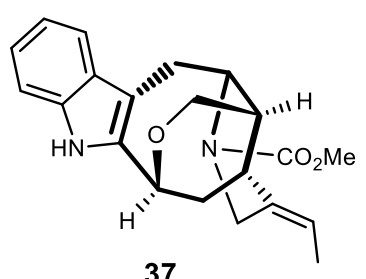

37

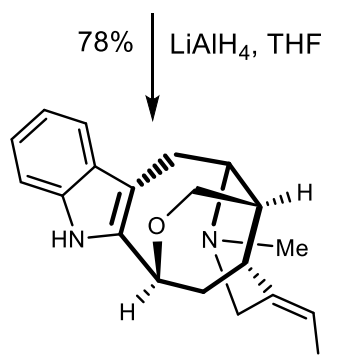

13: 19-Z-Taberpsychine

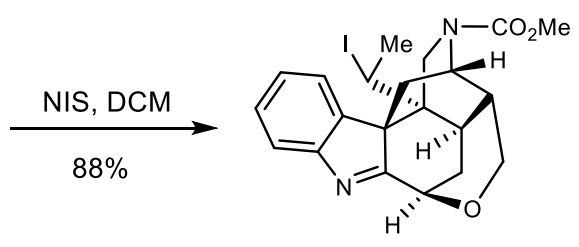

38

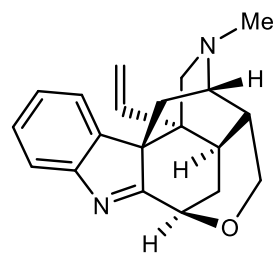

14: Koumine

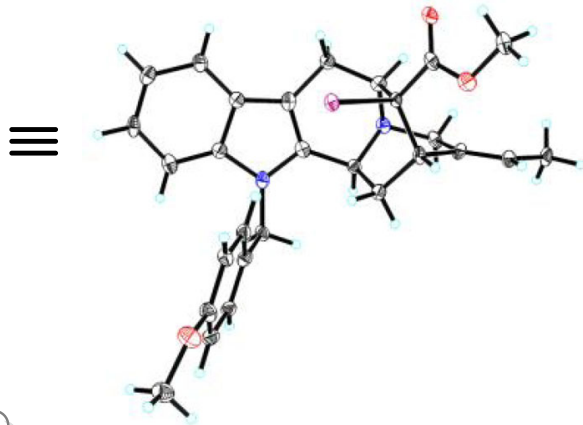

X-ray structure of $\mathbf{2 5 b}$

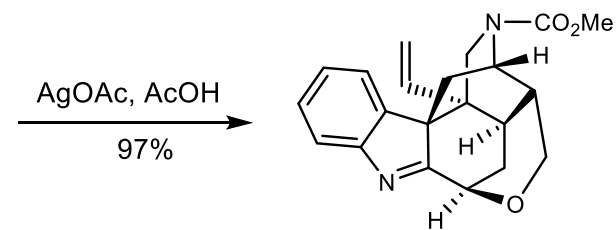

39 $79 \%\rfloor \mathrm{LiAlH}_{4}, \mathrm{THF}$

PhIO, DCM

Fig. 8 Total synthesis of koumine-type alkaloids. Key transformations are light induced radical reduction of iodide $\mathbf{2 5} \mathbf{b}$ and NIS mediated cyclization of $\mathbf{3 7}$ to access koumine-type alkaloids. [Ir(ppy) $)_{2}$ (dtbbpy)]PF 6 (4,4'-di-tert-butyl-2,2'-bipyridine)bis[(2-pyridinyl)phenyl]iridium(III) hexafluorophosphate, NIS $\mathrm{N}$-iodosuccinimide, DIPEA N,N-Diisopropylethylamine.

new compounds were characterized by IR, ${ }^{1} \mathrm{H}$ NMR, ${ }^{13} \mathrm{C}$ NMR, and HRMS. Silica gel (200-300 mesh) for column chromatography and silica $\mathrm{GF}_{254}$ for TLC were obtained from Merck Chemicals Co. Ltd. (Shanghai). Anhydrous THF was dried by distillation over metallic sodium and benzophenone; dichloromethane, pyridine, and methanol were distilled from calcium hydride. Starting materials and reagents used in reactions were obtained commercially from Acros, Aldrich, and Adamasbeta ${ }^{\circ}$, and were used without purification, unless otherwise indicated. All reactions were conducted in dried glassware under a positive pressure of dry nitrogen or argon. Reagents and starting materials were accordingly transferred via syringe or cannula. Reaction temperatures refer to the external oil bath temperature.

\section{Data availability}

The authors declare that the data supporting the findings of this study are available within the article and its Supplementary Information files. For the experimental procedures and spectroscopic and physical data of compounds, see Supplementary Methods. For ${ }^{1} \mathrm{H}$ and ${ }^{13} \mathrm{C}\left\{{ }^{1} \mathrm{H}\right\}$ NMR spectra of compounds, see Supplementary Figs. 1-109. For the comparisons of ${ }^{1} \mathrm{H}$ and ${ }^{13} \mathrm{C}$ NMR spectra of the natural and synthetic alkaloids, see Supplementary Tables $4-20$. For the X-ray crystallographic data of compounds 16a, 25, 1, 5, and 25b, see Supplementary Tables 21-25. The X-ray crystallographic coordinates for structures reported in this study have been deposited in the Cambridge Crystallographic Data Centre (16a: CCDC 2070256, 25: CCDC 2070257,
1: CCDC 2070258, 5: CCDC 2070259, and 25b: CCDC 2123071). These data can be obtained free of charge from The Cambridge Crystallographic Data Centre via www.ccdc.cam.ac.uk/data_request/cif.

Received: 21 June 2021; Accepted: 21 January 2022;

Published online: 17 February 2022

\section{References}

1. Lounasmaa, M., Hanhinen, P. \& Westersund, M. The Alkaloids: Chemistry and Biology (ed. Cordell, G. A.) 103-195 (Academic, San Diego, 1999).

2. Rahman, M. T. \& Cook, J. M. Studies in Natural Products Chemistry (ed. AttaUr-Rahman, F. R. S.) 1-57 (Elsevier, 2020).

3. Namjoshi, O. A. \& Cook, J. M. The Alkaloids: Chemistry and Biology (ed. Knölker, H.-J.) 63-169 (Academic, San Diego, 2016).

4. Wu, F.; Kerčmar, P., Zhang, C. \& Stöckigt, J. The Alkaloids: Chemistry and Biology (ed. Knölker, H.-J.) 1-61 (Academic, San Diego, 2016).

5. Takayama, H. \& Sakai, S.-I. Gelsemiurn alkaloids. The Alkaloids: Chemistry and Phamacology (ed. Cordell, G. A.) 1-78 (Academic, San Diego, 1997). 
6. Lounasmaa, M. \& Hanhinen, P. The Alkaloids: Chemistry and Biology (ed. Cordell, G. A.) 1-87 (Academic, San Diego, 2001).

7. Boğa, M., Bingül, M., Özkan, E. E. \& Şahin, H. Studies in Natural Products Chemistry (ed. Atta-Ur-Rahman, F. R. S.) 251-299 (Elsevier, 2019)

8. Liu, L., Cao, J.-X., Yao, Y.-C. \& Xu, S. P. Progress of pharmacological studies on alkaloids from Apocynaceae. J. Asian Nat. Prod. Res. 15, 166-184 (2013).

9. Stöckigt, J. The Alkaloids: Chemistry and Pharmacology (ed. Cordell, G. A.) 115-172 (Academic, San Diego, 1995).

10. Rosales, P. F., Bordin, G. S., Gower, A. E. \& Moura, S. Indole alkaloids: 2012 until now, highlighting the new chemical structures and biological activities. Fitoterapia 143, 104558 (2020).

11. Pan, L. et al. Bioactive indole alkaloids isolated from Alstonia angustifolia. Phytochem. Lett. 10, 54-59 (2014).

12. Keawpradub, N., Kirby, G., Steele, J. \& Houghton, P. Antiplasmodial activity of extracts and alkaloids of three alstonia species from Thailand. Planta Med. 65, 690-694 (1999).

13. Zeng, J. et al. Rauvomines A and B, two monoterpenoid indole alkaloids from Rauvolfia vomitoria. Org. Lett. 19, 3998-4001 (2017).

14. Zaima, K. et al. Vasorelaxant activity of indole alkaloids from Tabernaemontana dichotoma. J. Nat. Med. 67, 9-16 (2013).

15. Tan, S.-J. et al. Oxidized derivatives of macroline, sarpagine, and pleiocarpamine alkaloids from Alstonia angustifolia. J. Nat. Prod. 77, 2068-2080 (2014).

16. Guo, D. et al. Therapeutic effect of vinorine on sciatic nerve injured rat. Neurochem. Res. 43, 375-386 (2018).

17. Rahman, M. T., Tiruveedhula, V. V. \& Cook, J. M. Synthesis of bisindole alkaloids from the Apocynaceae which contain a macroline or sarpagine unit: a review. Molecules 21, 1525-1565 (2016).

18. Edwankar, C. R., Edwankar, R. V., Rallapalli, S. \& Cook, J. M. General approach to the total synthesis of macroline-related sarpagine and ajmaline alkaloids. Nat. Prod. Commun. 3, 1839-1870 (2008).

19. Lewis, S. E. Recent advances in the chemistry of macroline, sarpagine and ajmaline-related indole Alkaloids. Tetrahedron 62, 8655-8681 (2006).

20. Gerlinger, C. K. G. \& Gaich, T. Structure-pattern-based total synthesis. Chem. Eur. J. 25, 10782-18681 (2019).

21. Dagoneau, D., Wang, Q. \& Zhu, J. Towards the sarpagine-ajmaline-macroline family of indole alkaloids: Enantioselective synthesis of an $\mathrm{N}$-demethyl alstolactone diastereomer. Chem. Eur. J. 26, 4866-4873 (2020).

22. Zhao, Z. et al. Facile Synthesis of pyridines from propargyl amines: concise total synthesis of suaveoline alkaloids. Angew. Chem. Int. Ed. 58, 1148-1152 (2019).

23. Rahman, M. T., Deschamps, J. R., Imler, G. H. \& Cook, J. M. Total synthesis of sarpagine-related bioactive indole alkaloids. Chem. Eur. J. 24, 2354-2359 (2018).

24. Kitajima, M., Watanabe, K., Maeda, H., Kogure, N. \& Takayama, H. Asymmetric total synthesis of sarpagine-related indole alkaloids hydroxygardnerine, hydroxygardnutine, gardnerine, (E)-16-epi-normacusine B, and koumine. Org. Lett. 18, 1912-1915 (2016).

25. Edwankar, C. R., Edwankar, R. V., Deschamps, J. R. \& Cook, J. M. Natureinspired stereospecific total synthesis of $\mathrm{P}-(+)$-dispegatrine and four other monomeric sarpagine indole alkaloids. Angew. Chem. Int. Ed. 51, 11762-11765 (2012).

26. van Tamelen, E. E. \& Oliver, L. K. The biogenetic-type total synthesis of ajmaline. J. Am. Chem. Soc. 92, 2136-2137 (1970).

27. Masamune, S. et al. The synthesis of ajmaline. J. Am. Chem. Soc. 89 , 2506-2507 (1967).

28. Cox, P., Craig, D., Ioannidis, S. \& Rahn, V. S. 4-(Phenylsulfonyl)-4lithiocyclopentene as a nucleophilic 2-pentene-1,5-dial synthetic equivalent. An aziridine-based synthetic approach to (-)-alstonerine. Tetrahedron Lett. 46, 4687-4690 (2005).

29. Kadam, V. D. et al. Stereoselective access to the core structure of macrolinetype indole alkaloids: Total synthesis of macroline and alstomicine. Org. Lett. 20, 4782-4786 (2018).

30. Zhang, L., Zhang, Y., Li, W. \& Qi, X. Total synthesis of (-)-alstofolinine A through a furan oxidation/rearrangement and indole nucleophilic cyclization cascade. Angew. Chem. Int. Ed. 58, 4988-4991 (2019).

31. Zhang, L.-H. \& Cook, J. M. Pictet-Spengler reactions in aprotic media: $N_{\mathrm{b}^{-}}$ Benzyl promoted retention of optical activity in the synthesis of an indolo substituted azabicyclo[3.3.1]nonane, a key template for the synthesis of macroline alkaloids. Heterocycles 27, 2795-2802 (1988).

32. Trudell, M. L. \& Cook, J. M. Total synthesis of ( \pm -suaveoline. J. Am. Chem. Soc. 111, 7504-7507 (1989).

33. Bi, Y., Zhang, L.-H., Hamaker, L. K. \& Cook, J. M. Enantiospecific synthesis of $(-)$-alstonerine and $(+)$-macroline as well as a partial synthesis of (+)-villalstonine. J. Am. Chem. Soc. 116, 9027-9041 (1994).

34. Li, J. et al. General approach for the synthesis of ajmaline/sarpagine indole alkaloids: Enantiospecific total synthesis of $(+)$-ajmaline, alkaloid G, and norsuaveoline via the asymmetric Pictet-Spengler reaction. J. Am. Chem. Soc. 121, 6998-7010 (1999).
35. Yu, J., Wearing, X. Z. \& Cook, J. M. Stereocontrolled total synthesis of (-)-vincamajinine and (-)-11-methoxy-17-epivincamajine. J. Am. Chem. Soc. 126, 1358-1359 (2004).

36. Yu, J., Wearing, X. Z. \& Cook, J. M. A general strategy for the synthesis of vincamajine-related indole alkaloids: Stereocontrolled total synthesis of $(+)$-dehydrovoachalotine, (-)-vincamajinine, and (-)-11-methoxy-17epivincamajine as well as the related quebrachidine diol, vincamajine diol, and vincarinol. J. Org. Chem. 70, 3963-3979 (2005).

37. Edwankar, R. V., Edwankar, C. R., Deschamps, J. \& Cook, J. M. Regiospecific, enantiospecific total synthesis of C-19 methyl substituted sarpagine alkaloids dihydroperaksine-17-al and dihydroperaksine. Org. Lett. 13, 5216-5219 (2011).

38. Rahman, M. T., Deschamps, J. R., Imler, G. H., Schwabacher, A. W. \& Cook, J. $\mathrm{M}$. Total synthesis of macrocarpines D and $\mathrm{E}$ via an enolate-driven coppermediated cross-coupling process: Replacement of catalytic palladium with copper iodide. Org. Lett. 18, 4174-4177 (2016).

39. Stephen, M. R. et al. Concise total synthesis of (-)-affinisine oxindole, $(+)$-isoalstonisine, $(+)$-alstofoline, $(-)$-macrogentine, $(+)-N_{\mathrm{a}}-$ demethylalstonisine, (-)-alstonoxine A, and (+)-alstonisine. Chem. Eur. J. 23, 15805-15819 (2017)

40. Rahman, M. T. \& Cook, J. M. Unprecedented stereocontrol in the synthesis of 1,2,3-trisubstituted tetrahydro- $\beta$-carbolines through an asymmetric Pictet-Spengler reaction towards sarpagine-type indole alkaloids. Eur. J. Org. Chem. 2018, 3224-3229 (2018).

41. Rahman, M. T. \& Cook, J. M. The ambidextrous Pictet-Spengler reaction: Access to the (+)- or (-)-enantiomers of the bioactive C-19 methyl-substituted sarpagine/macroline/ajmaline alkaloids from either D- or L-tryptophan. Synthesis 51, 1980-1988 (2019).

42. Rahman, M. T., Namjoshi O. A. \& Cook, J. M. Progress in Heterocyclic Chemistry (ed. Gribble, G. W. \& Joule, J. A.) 32, 1-26 (Elsevier, 2021).

43. Bailey, P. D., Clingan, P. D., Mills, T. J., Price, R. A. \& Pritchard, R. G. Total synthesis of (-)-raumacline. Chem. Commun. 2003, 2800-2801 (2003).

44. Bailey, P. D. et al. Stereoselectivity and conformational control in the synthesis of ajmaline and epi-ajmaline alkaloids. Tetrahedron Lett. 54, 1726-1729 (2013).

45. Miller, K. A. \& Martin, S. F. Concise, Enantioselective total synthesis of (-)-alstonerine. Org. Lett. 9, 1113-1116 (2007).

46. Neipp, C. E. \& Martin, S. F. Synthesis of bridged azabicyclic structures via ring-closing olefin metathesis. J. Org. Chem. 68, 8867-8878 (2003)

47. Kuethe, J. T., Wong, A., Davies, I. W. \& Reider, P. J. Aza-Diels-Alder/ intramolecular Heck cyclization approach to the tetrahydro- $\beta$-carboline skeleton of the ajmaline/sarpagine alkaloids. Tetrahedron Lett. 43, 3871-3874 (2002).

48. Tan, Q. et al. Copper-catalyzed aerobic oxidative cyclization cascade to construct bridged skeletons: Total synthesis of $(-)$-suaveoline. Angew. Chem. Int. Ed. 58, 6420-6424 (2019).

49. Yang, Z. et al. Asymmetric total synthesis of sarpagine and koumine alkaloids Angew. Chem. Int. Ed. 60, 13105-13111 (2021).

50. Ohba, M. \& Natsutani, I. Total synthesis of the proposed structure of macrocaffrine. Tetrahedron 63, 12689-12694 (2007).

51. Krüger, S. \& Gaich, T. Enantioselective, protecting-group-free total synthesis of sarpagine alkaloids-a generalized approach. Angew. Chem. Int. Ed. 54 315-317 (2015).

52. Krüger, S. \& Gaich, T. Total syntheses of vellosimine, $N$-methylvellosimine, and 10-methoxyvellosimine and formal synthesis of 16-epinormacusine B through a [5+2] cycloaddition. Eur. J. Org. Chem. 2016, 4893-4899 (2016).

53. Rebmann, H., Gerlinger, C. K. G. \& Gaich, T. Gram-scale total synthesis of sarpagine alkaloids and non-natural derivatives. Chem. Eur. J. 25, 2704-2707 (2019).

54. Tran, Y. S. \& Kwon, O. An application of the phosphine-catalyzed $[4+2]$ annulation in indole alkaloid synthesis: formal syntheses of $( \pm)$-alstonerine and ( \pm )-macroline. Org. Lett. 7, 4289-4291 (2005).

55. Gorman, M., Burlingame, A. \& Biemann, K. Application of mass spectrometry to structure problems. The structure of quebrachidine. Tetrahedron Lett. 4, 39-46 (1963).

56. Arai, $\mathrm{H}$. et al. Alstiphyllanines I-O, ajmaline type alkaloids from Alstonia macrophylla showing vasorelaxant activity. Bioorg. Med. Chem. 20, 3454-3459 (2012).

57. Zhang, H. Syntheses of bioactive natural products and natural-product-like compounds based on their key structural units. Synlett 25, 1953-1970 (2014)

58. Chen, W. et al. Total synthesis of (-)-vindoline. Tetrahedron 75, 1751-1759 (2019).

59. Chen, W. et al. Total synthesis of (-)-vindorosine. Angew. Chem. Int. Ed. 56, 12327-12331 (2017)

60. Xi, Y.-K. et al. Total synthesis of spirotryprostatins through organomediated intramolecular umpolung cyclization. Chem. Eur. J. 25, 3005-3009 (2019).

61. Chen, W. et al. Iodine mediated deprotection of $N$-tertbutanesulfinyl amines: a functional group compatible method. Chem. Commun. 50, 6259-6262 (2014). 
62. Wang, M.-S., Wang, Z., Chen, W., Yang, X. \& Zhang, H. Synthesis of oxabridged medium-sized carbocyclic rings via Prins cyclization. Org. Lett. 21, 1881-1884 (2019).

63. Sun, Y. et al. Bioinspired total synthesis of sespenine. Angew. Chem. Int. Ed. 53, 9012-9016 (2014).

64. Sun, Y., Li, R., Zhang, W. \& Li, A. Total synthesis of indotertine A and drimentines A, F, and G. Angew. Chem. Int. Ed. 52, 9201-9204 (2013).

65. Davis, F. A., Melamed, J. Y. \& Sharik, S. S. Total synthesis of (-)-normalindine via addition of metalated 4-methyl-3-cyanopyridine to an enantiopure sulfinimine. J. Org. Chem. 71, 8761-8766 (2006).

66. More, J. D. \& Finney, N. S. A Simple and advantageous protocol for the oxidation of alcohols with $o$-iodoxybenzoic Acid (IBX). Org. Lett. 4, 3001-3003 (2002).

67. Cacchi, S., Morera, E. \& Ortar, G. Palladium-catalyzed reduction of enol triflates to alkenes. Tetrahedron Lett. 25, 4821-4824 (1984).

68. Keverline, K. I., Abraham, B., Lewin, A. H. \& Carroll, F. I. Synthesis of $2 \beta, 3 \alpha-$ and $2 \beta, 3 \beta$-isomers of 3 -( $p$-substituted phenyl)-tropane-2-carboxylic acid methyl esters. Tetrahedron Lett. 36, 3099-3102 (1995).

69. Dounay, A. B., Humphreys, P. G., Overman, L. E. \& Wrobleski, A. D. Total synthesis of the strychnos alkaloid (+)-minfiensine: Tandem enantioselective intramolecular Heck-iminium ion cyclization. J. Am. Chem. Soc. 130, 5368-5377 (2008).

70. Zu, L., Boal, B. W. \& Garg, N. K. Total synthesis of ( \pm )-aspidophylline A. J. Am. Chem. Soc. 133, 8877-8879 (2011).

71. Ren, W., Wang, Q. \& Zhu, J. Total synthesis of ( \pm )-aspidophylline A. Angew. Chem. Int. Ed. 53, 1818-1821 (2014).

72. Ren, W., Wang, Q. \& Zhu, J. Total synthesis of ( \pm )-strictamine. Angew. Chem. Int. Ed. 55, 3500-3503 (2016).

73. Teng, M., Zi, W. \& Ma, D. Total Synthesis of the monoterpenoid indole alkaloid ( \pm )-aspidophylline A. Angew. Chem. Int. Ed. 53, 1814-1817 (2014).

74. Li, G., Xie, X. \& Zu, L. Total synthesis of calophyline A. Angew. Chem. Int. Ed. 55, 10483-10486 (2016).

75. Wang, T. et al. Asymmetric total synthesis of (-)-aspidophylline A. Org. Lett. 19, 1650-1653 (2017).

76. $\mathrm{Li}, \mathrm{W}$. et al. Asymmetric total syntheses of the akuammiline alkaloids (-)-strictamine and (-)-rhazinoline. Angew. Chem. Int. Ed. 58, 6059-6063 (2019).

77. Yu, J. et al. General approach for the synthesis of sarpagine indole alkaloids. Enantiospecific total synthesis of $(+)$-vellosimine, $(+)$-normacusine $\mathrm{B}$, (-)-alkaloid $\mathrm{Q}_{3},(-)$-panarine, $(+)-N_{\mathrm{a}}$-methylvellosimine, and (+)- $N_{\mathrm{a}}$-methyl16-epipericyclivine. J. Org. Chem. 68, 7565-7581 (2003).

78. Jensen, C. M. et al. Can decarbonylation of acyl radicals be overcome in radical addition reactions? En route to a solution employing $\mathrm{N}$-acyl oxazolidinones and $\mathrm{SmI}_{2} / \mathrm{H}_{2} \mathrm{O}$. J. Am. Chem. Soc. 127, 6544-6545 (2005).

79. Hansen, A. M. et al. Mechanistic evidence for intermolecular radical carbonyl additions promoted by samarium diiodide. J. Am. Chem. Soc. 128, 9616-9617 (2006).

80. Karaffa, J., Lindsay, K. B. \& Skrydstrup, T. Expanding the scope of the acyltype radical addition reactions promoted by $\mathrm{SmI}_{2}$. J. Org. Chem. 71, 8219-8226 (2006).

81. Mittag, T., Christensen, K. L., Lindsay, K. B., Nielsen, N. C. \& Skrydstrup, T. Direct entry to peptidyl ketones via $\mathrm{SmI}_{2}$-mediated $\mathrm{C}-\mathrm{C}$ bond formation with readily accessible $N$-peptidyl oxazolidinones. J. Org. Chem. 73, 1088-1092 (2008).

82. Taaning, R. H. et al. $\mathrm{SmI}_{2}$-promoted intra- and intermolecular C-C bond formation with chiral $N$-acyl oxazolidinones. Tetrahedron 64, 11884-11895 (2008).

83. Taaning, R. H., Lindsay, K. B., Schiott, B., Daasbjerg, K. \& Skrydstrup, T. Importance of C-N bond rotation in $\mathrm{N}$-acyl oxazolidinones in their $\mathrm{SmI}_{2}$ promoted coupling to acrylamides. J. Am. Chem. Soc. 131, 10253-10262 (2009).

84. Mittag, T., Otzen, D. E., Nielsen, N. C. \& Skrydstrup, T. Synthesis of a ketomethylene isostere of the fibrillating peptide SNNFGAILSS. J. Org. Chem. 74, 7955-7957 (2009).

85. Crawford, J. J. et al. Use of a highly effective intramolecular Pauson-Khand cyclisation for the formal total synthesis of $( \pm)$ - $\alpha$ - and $\beta$-cedrene by preparation of cedrone. Tetrahedron 62, 11360-11370 (2006)

86. Bjedov, S., Jakimov, D., Pilipović, A., Poša, M. \& Sakač, M. Antitumor activity of newly synthesized oxo and ethylidene derivatives of bile acids and their amides and oxazolines. Steroids 120, 19-25 (2017).

87. Zavesky, B. P., Cruz, P. D. J. \& Johnson, J. S. Progress toward a convergent, asymmetric synthesis of jervine. Org. Lett. 22, 3537-3541 (2020).

88. Ando, K. \& Takama, D. Stereoselective synthesis of trisubstituted ( $Z$ )-alkenes from ketones via the Julia-Kocienski olefination using 1-methyl- and 1-tertbutyl-1H-tetrazol-5-yl alkyl sulfones. Org. Lett. 22, 6907-6910 (2020).

89. Sakai, S. et al. Indole alkaloids isolated from Gelsemium Elegans (Thailand) 19-(Z)-akuammidine, 16-epi-voacarpine, 19-hydroxydihydrogelsevirine, and the revised structure of koumidine. Chem. Pharm. Bull. 35, 4668-4671 (1987).
90. Ponglux, D. et al. Studies on the indole alkaloids of Gelsemium Elegans (Thailand): Structure elucidation and proposal of biogenetic route. Tetrahedron 44, 5075-5094 (1988).

91. Yin, W., Ma, J., Rivas, F. M. \& Cook, J. M. First Enantiospecific total synthesis of the important biogenetic intermediates, $(+)$-polyneuridine and $(+)$-polyneuridine aldehyde, as well as 16-epi-vellosimine and macusine A Org. Lett. 9, 295-298 (2007)

92. Yin, W. et al. Enantiospecific total synthesis of the important biogenetic intermediates along the ajmaline pathway, $(+)$-polyneuridine and (+)-polyneuridine aldehyde, as well as 16-epivellosimine and macusine A. J. Org. Chem. 75, 3339-3349 (2010).

93. Yuldashev, P. K. \& Yunusov, S. Y. The structure of vincarine. Khim. Prir. Soedin. 1, 110-113 (1965).

94. Jokela, R. \& Lounasma, M. A ${ }^{1} \mathrm{H}$ - and ${ }^{13} \mathrm{C}-\mathrm{NMR}$ study of seven ajmaline-type alkaloids. Planta Med. 62, 577-579 (1996).

95. Turpin, V., Poupon, E., Jullian, J.-C. \& Evanno, L. Biosynthetically relevant reactivity of polyneuridine aldehyde. Eur. J. Org. Chem. 2020, 6989-6991 (2020).

96. Ahamada, K., Benayad, S., Poupon, E. \& Evanno, L. Polyneuridine aldehyde: structure, stability overviews and a plausible origin of flavopereirine. Tetrahedron Lett. 57, 1718-1720 (2016).

97. Zhang, W. et al. Total synthesis of (+)-haperforin G. J. Am. Chem. Soc. 142, 19487-19492 (2020)

98. Kitajima, M., Takayama, H. \& Sakai, S.-I. Stereoselective transformation of ajmaline into three minor Gelsemium alkaloids, koumidine, (19Z)anhydrovobasinediol [(19Z)-taberpsychine] and $N$-demethoxyrankinidine and their absolute configuration. J. Chem. Soc. Perkin Trans. 1, 1773-1779 (1991)

99. Magnus, P., Mugrage, B., DeLuca, M. R. \& Cain, G. A. Studies on Gelsemium alkaloids. Total synthesis of $(+)$-koumine, $(+)$-taberpsychine, and (+)-koumidine. J. Am. Chem. Soc. 112, 5220-5230 (1990).

100. Kerkovius, J. K. \& Kerr, M. A. Total synthesis of isodihydrokoumine, (19Z)-taberpsychine, and (4R)-isodihydroukoumine $N_{4}$-Oxide. J. Am. Chem. Soc. 140, 8415-8419 (2018).

101. Liu, Z.-J. \& Yu, Q.-S. A partial synthesis of koumine. Youji Huaxue 1, 36-37 (1986)

102. Sakai, S. et al. Biomimetic synthesis of koumine skeleton: Partial synthesis of 11-methoxykoumine (gelsemium-type alkaloid) from 18-hydroxygardnerine. Tetrahedron Lett. 27, 4585-4588 (1986).

103. Zhang, W. et al. Five new koumine-type alkaloids from the roots of Gelsemium elegans. Fitoterapia 118, 112-117 (2017).

104. Liu, C.-T., Wang, Q.-W. \& Wang, C.-H. Structure of koumine. J. Am. Chem. Soc. 103, 4634-4635 (1981).

\section{Acknowledgements}

This work was supported by grants from Natural Science Foundation of China (21572197, U1702286, 21762047, and 21901224), the Program for Changiiang Scholars and Innovative Research Team in University (IRT17R94), Ling-Jun Scholars of Yunnan Province (202005AB160003), YunLing Scholar Programs, Yunnan Fundamental Research Projects (2019FI018), Talent Plan of Yunnan Province (YNWR-QNBJ-2018025) and grant from the National Key Research and Development Program of China (2019YFE0109200). We thank Prof. Hiroshi Morita of Hoshi University for providing the authentic spectra of alstiphyllanine J for comparison. Dr. Xiaonian Li in Kunming Institute of Botany is gratefully acknowledged for X-ray crystallography analysis.

\section{Author contributions}

H.Z. conceived the synthetic design, and X.Y. and H.Z. directed the project. W.C., Y.M. W.H., Y.W., Y.H., Y.Z., H.T., and K.W. conducted the experimental work and data analysis. H.Z. and W.C. wrote the manuscript.

\section{Competing interests}

The authors declare no competing interests.

\section{Additional information}

Supplementary information The online version contains supplementary material available at https://doi.org/10.1038/s41467-022-28535-x.

Correspondence and requests for materials should be addressed to Xiaodong Yang or Hongbin Zhang.

Peer review information Nature Communications thanks the anonymous reviewer(s) for their contribution to the peer review of this work.

Reprints and permission information is available at http://www.nature.com/reprints

Publisher's note Springer Nature remains neutral with regard to jurisdictional claims in published maps and institutional affiliations. 
(c) (i) Open Access This article is licensed under a Creative Commons Attribution 4.0 International License, which permits use, sharing, adaptation, distribution and reproduction in any medium or format, as long as you give appropriate credit to the original author(s) and the source, provide a link to the Creative Commons license, and indicate if changes were made. The images or other third party material in this article are included in the article's Creative Commons license, unless indicated otherwise in a credit line to the material. If material is not included in the article's Creative Commons license and your intended use is not permitted by statutory regulation or exceeds the permitted use, you will need to obtain permission directly from the copyright holder. To view a copy of this license, visit http://creativecommons.org/ licenses/by/4.0/.

(C) The Author(s) 2022 